\title{
Hibiscus chlorotic ringspot virus p27 and Its Isoforms Affect Symptom Expression and Potentiate Virus Movement in Kenaf (Hibiscus cannabinus L.)
}

\author{
T. Zhou, ${ }^{1,2}$ Z. F. Fan, ${ }^{1,2}$ H. F. Li, ${ }^{1}$ and S. M. Wong ${ }^{3,4}$ \\ ${ }^{1}$ Department of Plant Pathology and ${ }^{2}$ State Key Laboratory of Agrobiotechnology, China Agricultural University, Beijing \\ 100094, China; ${ }^{3}$ Temasek Life Sciences Laboratory, 1 Research Link, 117604, Singapore; ${ }^{4}$ Department of Biological \\ Sciences, National University of Singapore, 14 Science Drive 4, Kent Ridge, 117543, Singapore
}

Submitted 22 November 2005. Accepted 28 March 2006.

\begin{abstract}
Hibiscus chlorotic ringspot virus (HCRSV), a member of the genus Carmovirus, encodes p27 (27-kDa protein) and two other in-frame isoforms (p25 and p22.5) that are coterminal at the carboxyl end. Only p27, which initiates at the ${ }^{2570}$ CUG codon, was detected in transfected kenaf (Hibiscus cannabinus L.) protoplasts through fusion to a Flag tag at either its $\mathbf{N}$ or $\mathbf{C}$ terminus. Subcellular localization of a p27-green fluorescent fusion protein in kenaf epidermal cells showed that it was localized to membrane structures close to cell walls. To study the functions of these proteins, a number of start codon mutants and premature translation termination mutants were constructed. Phenotypic differences were observed between the wild-type virus and these mutants during infection. Infectivity assays on plants indicated that p27 is a determinant of symptom severity. Without p25, appearance of symptoms on systemically infected kenaf leaves was delayed by 4 to 8 days. In a timecourse analysis, Western blot assays revealed that the delay corresponded to retardation in virus systemic movement, which suggested that p25 is probably involved in virus systemic movement. Mutations disrupting expression of p22.5 did not affect symptoms or virus movement.
\end{abstract}

Additional keywords: transmembrane domain, noncanonical start codon.

Hibiscus chlorotic ringspot virus (HCRSV) is a positivestrand RNA virus that is a member of the genus Carmovirus. All carmoviruses have a genome organization similar to Carnation mottle virus (Brunt et al. 1996). The HCRSV genomic RNA (gRNA) is 3,911 nucleotides (nt) and possesses a t-RNAlike structure at the $3^{\prime}$ terminus. The genome (Fig. 1) has the potential to encode seven open reading frames (ORF). The two $5^{\prime}$ proximal ORF encode p28 and its readthrough p81, which probably are involved in virus replication, based on data from other carmoviruses (Hacker et al. 1992; Rajendran et al. 2002; White et al. 1995). Two overlapping small centrally located ORF encode $\mathrm{p} 8$ and $\mathrm{p} 9$, which are translated from subgenomic RNA1 (sgRNA1). By comparison with Turnip crinkle virus (TCV) (Hacker et al. 1992; Li et al. 1998), HCRSV p8 and p9 may be involved in cell-to-cell movement. The coat protein (CP) p38, which is encoded by sequences in the $3^{\prime}$ region of

Corresponding authors: S. M. Wong; Telephone: +65 65162976; E-mail: dbswsm@nus.edu.sg; and H. F. Li; Telephone: +86 10 62732771; E-mail: plpath@cau.edu.cn the genome and is translated from sgRNA2, is required for virus encapsidation (Huang et al. 2000). The transcriptional start sites of sgRNA1 and sgRNA2 are located at nt 2,178 and 2,438, respectively (Li and Wong 2006). Two novel ORF, p23 and $\mathrm{p} 25$, were identified in an in vitro translation system from wheat germ extracts (Huang et al. 2000). ORF p23 is located in the $5^{\prime}$ region and overlaps the p28 ORF. The p23 protein is required for replication of HCRSV in kenaf (Liang et al. 2002b). ORF for p25 and p22.5 are located near the $3^{\prime}$ terminal region of the genome and their start codons are located at nt 2,603 and 2,666, respectively. A new ORF for p27 is initiated at the noncanonical start codon ${ }^{2570} \mathrm{CUG}$ and is translated from sgRNA2 (D. C. Y. Koh, unpublished data).

In this study, we have investigated p27 and its isoforms for their i) presence in vivo, ii) possible functions through mutations abolishing the expression of p27, p25 and p22.5, and iii) influence on phenotypes and systemic symptom development. In addition, the subcellular localization of a p27- GFP (green fluorescent protein) fusion protein was examined in kenaf leaves.

\section{RESULTS}

Expression of p27 in transfected kenaf protoplasts and prediction of its potential structure domain.

To detect expression of p27 and its isoforms in vivo, three constructs, p27 ${ }_{\mathrm{N}}$ Flag, p27 $\mathrm{C}$ Flag, and p27atgFlag, were made (Fig. 2A). Constructs p27 ${ }_{\mathrm{N}}$ Flag and p27 $\mathrm{C}$ Flag express a FLAG epitope at the $\mathrm{N}$ or $\mathrm{C}$ terminus of $\mathrm{p} 27$, under the control of the ${ }^{2570} \mathrm{CUG}$ start codon. The p27atgFlag construct expresses a FLAG epitope at the $\mathrm{N}$ terminus of $\mathrm{p} 27$, which is under the control of a start codon mutated from ${ }^{2570} \mathrm{CUG}$ to ${ }^{2570} \mathrm{AUG}$. RNA transcripts from the three constructs replicate normally in transfected kenaf protoplasts (Fig. 2B), but they are not infectious on whole kenaf plants, because the mutants affected the expression of either $\mathrm{p} 9$ or the $\mathrm{CP}$ (data not shown). Total proteins obtained from $1 \times 10^{5}$ transfected kenaf protoplasts with polyethylene glycol (PEG) buffer (mock transfection), p223, p27 ${ }_{\mathrm{N}}$ Flag, p27 $\mathrm{C}$ Flag, and p27atgFlag at $72 \mathrm{~h}$ posttransfection (hpt) were probed with an anti-FLAG M2 monoclonal antibody (mAb). A specific protein with a molecular weight of $27 \mathrm{kDa}$ was detected at much lower levels for p27 $\mathrm{N}$ Flag and p27 $\mathrm{C}$ Flag than for p27atgFlag transfected protoplasts (Fig. 2C). The results showed that the FLAG-tagged p27 accumulated to a low level in HCRSV transfected protoplasts. The band was absent in protoplasts transfected with the wild-type virus or mocktransfected protoplasts. 
Using the values assigned to each of the amino-acid residues to the Kyte-Doolittle $x-1$ values and a window size of 30 , the hydropathy plots show that HCRSV p27 has a highly hydrophobic domain at the C-terminal amino acids 198 to 220 (Fig. $3 \mathrm{~A})$. The program TMHMM version 2.0 predicts one transmembrane helix between amino acids 198 to 220 in p27 (Fig. 3B). It also predicts that the N-terminal 1 to 197 amino acids are located on the exterior of the membrane and the C-terminal amino acids 221 to 235 are located in the cytoplasm.

\section{Subcellular localization of the p27-GFP fusion protein.}

To confirm the predicted transmembrane property and probe the function of p27, the subcellular distribution of p27 was assessed with a p27-GFP fusion protein-expressing construct
(Fig. 4A) at various times after agroinfiltration. Results show that free GFP fluoresces in both the nuclei and the cytoplasm of the infiltrated leaf cells (Fig. 4Ba). In contrast, the p27-GFP fusion protein localized to membrane structures close to the cell walls (Fig. 4Bb) and no fluorescence was present in the cytoplasm or nuclei. In addition, strong fluorescence foci suggest that the p27-GFP fusion protein may be targeted to the plasmodesmata (Fig. 4Bb and c, arrows). Unexpectedly, many cell clusters developed green fluorescence in leaves infiltrated with the p27-GFP fusion construct (Fig. 4Bd). In contrast, free GFP was present in isolated single epidermal cells. More intensive quantitative counting of fluorescent loci also confirmed the presence of cluster of cells expressing the p27-GFP fusion protein (Table 1).

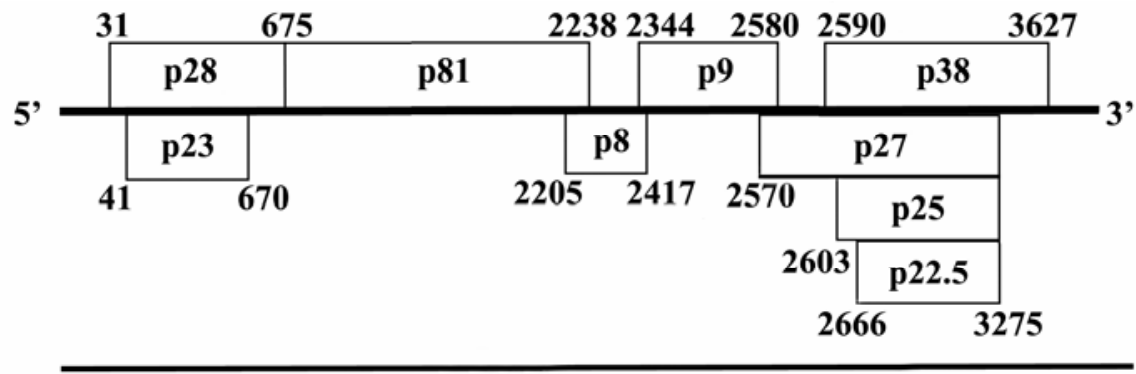

3911

gRNA

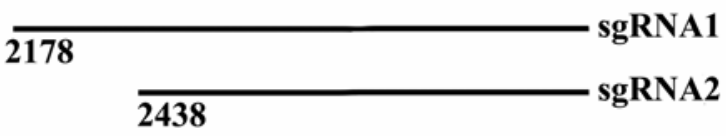

Fig. 1. Schematic representation (not drawn to scale) of Hibiscus chlorotic ringspot virus genome and its two subgenomic (sg)RNAs. Rectangles represent open reading frames assigned based on predicted molecular weight. The transcriptional sites of sgRNA1 and sgRNA2 are located at nucleotides 2,178 and 2,438 , respectively.

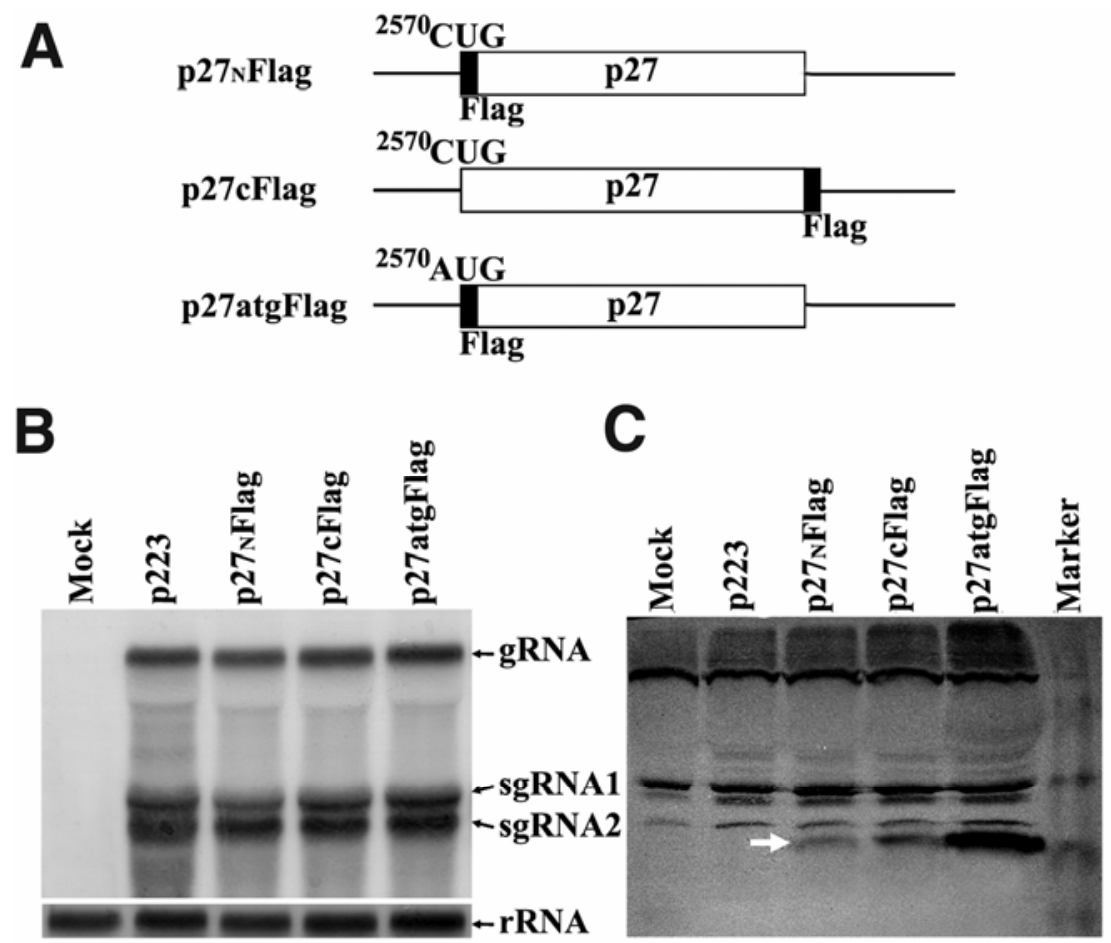

Fig. 2. Detection of p 27 in transfected kenaf protoplasts probed with anti-FLAG M2 monoclonal antibody. A, The constructs for p27 detection. FLAG epitope was fused either immediately after the start codon ${ }^{2570} \mathrm{CUG}$ in construct p27 $\mathrm{N}$ Flag, before the stop codon ${ }^{3275} \mathrm{UAA}$ in construct p27 $\mathrm{C}$ Flag, or the start codon mutated from ${ }^{2570} \mathrm{CUG}$ to ${ }^{2570} \mathrm{AUG}$ in construct p27atgFlag. The solid bar represents the engineered FLAG coding sequence. For simplicity, only the p27 open reading frame was shown. B, Northern blot showed that the three constructs could replicate as normally as wild type (p223) in kenaf protoplasts at $24 \mathrm{~h}$ after transfection. "Mock" represents RNA from protoplasts transfected with $40 \%$ polyethylene glycol (PEG) buffer. C, Immunodetection of p27 with anti-FLAG M2 monoclonal antibody that was prepared at $72 \mathrm{~h}$ after transfection with $40 \%$ PEG buffer (Mock), wild type Hibiscus chlorotic ringspot virus (HCRSV) (p223), p27 Flag, p27 Flag, and p27atgFlag, respectively. Arrow indicates the detected HCRSV p27. 
The p27N197-GFP construct, which expressed the N-terminal 197 amino acids of p27 without the transmembrane domain fused with a GFP, had similar distributions of green fluorescence in transfected cells (Fig. 4Be; Table 1). This result showed that the transmembrane domain was essential for specific localization of p27-GFP to membrane structures. As a negative control, the distribution of green fluorescence of the p38-GFP fusion protein was observed to be similar to that of the free GFP (Fig. 4Bf; Table 1). Membrane-specific localization was also confirmed by colocalization of the dye FM4-64 with p27-GFP (Fig. 4Bg, h, and i). These results provide further evidence for membrane association and plasmodesmatal targeting of the p27-GFP protein.

\section{p27 mutants attenuate symptom expression and reduce virulence.}

To determine the function of p27 in virus movement, symptom expression, and virulence of HCRSV, the start codon ${ }^{2570} \mathrm{CUG}$ of the HCRSV p27 ORF was mutated to either CCG or CAG. To create a truncated p27, ${ }^{2582} \mathrm{CAA}$ was mutated to ${ }^{2582} \mathrm{UAA}$, resulting in a premature stop codon. This led to abolition of the CUG initiation codon and premature termination of the p27, respectively. Three mutants, designated p27CCG, p27CAG, and p27trunc, respectively (Fig. 5), did not affect amino-acid residues in the overlapping $\mathrm{p} 9$ or the CP ORF.

Compared with the wild-type p223, the three mutants induced smaller chlorotic lesions on inoculated leaves 4 to 6 days postinoculation (dpi) (Fig. 6, B versus C) and the infection loci did not expand, even up to $18 \mathrm{dpi}$. On systemically infected leaves, the symptoms induced by the p27CCG, p27CAG, and p27trunc mutants were attenuated, showing mild mottling, vein-banding, and mild mosaic (Fig. 6F).

The progeny virus of each mutant was propagated, and there were no reversions or mutations, as confirmed by reverse transcription-polymerase chain reaction (RT-PCR) and sequencing of RNA extracted from systemically infected leaves. Western blot assays with anti-HCRSV CP antiserum showed that the level of CP accumulation for the mutants was comparable to that of the wild-type infection in the systemically infected leaves (Fig. 7A). Since reduced symptom severity was observed in all p27 start-codon mutants and the p27trunc mutant, p27 is believed to be a virulence determinant of HCRSV in kenaf.

\section{Alteration of ${ }^{2570} \mathrm{CUG}$ to ${ }^{2570} \mathrm{AUG}$ abolishes} virus cell-to-cell movement and expression of HCRSV CP.

Unexpectedly, three separate experiments revealed that none of the 42 test plants inoculated with mutant p27ATG, which has the ${ }^{2570}$ CUG mutated to the ${ }^{2570} \mathrm{AUG}$ start codon (Fig. 5), developed symptoms on inoculated or uninoculated leaves (Fig. 6A and D). In addition, no viral RNA was detected in inoculated leaves (Fig. 8A).

Kenaf protoplasts were used to examine whether the mutant p27ATG could replicate and express CP normally. The results indicated that the levels of the gRNA and sgRNAs of the mutants were comparable to those of the wild-type p223 (Fig. $8 \mathrm{~B})$. However, no detectable levels of the $\mathrm{CP}(\mathrm{p} 38)$ were present in p27ATG transfected kenaf protoplasts (Fig. 7B). Since the mutant p27ATG was able to replicate in kenaf protoplasts but could not accumulate in inoculated leaves, this suggests that the ${ }^{2570} \mathrm{CUG}$ to ${ }^{2570} \mathrm{AUG}$ mutation affected $\mathrm{CP}$ accumulation, which interfered with cell-to-cell movement.

\section{The p25 start codon mutant delays onset of systemic symptoms.}

To evaluate possible functions of the two isoforms of $\mathrm{p} 27$, the mutants p25ACG and p22.5ACG were constructed to alter the ${ }^{2603} \mathrm{AUG}$ and ${ }^{2666} \mathrm{AUG}$ to ${ }^{2603} \mathrm{ACG}$ and ${ }^{2666} \mathrm{ACG}$ (Fig. 5), respectively. The mutated sites did not change the amino-acid residues of the overlapping $\mathrm{CP}$. No difference was observed in symptom expression on the leaves inoculated with wild-type virus (Fig. 6B and $\mathrm{E}$ ) versus the two mutants. However, the onset of systemic symptoms with the p25ACG mutant was delayed for 4 to 8 days, whereas the p22.5ACG mutant elicited systemic symptoms at the same time as the wild-type virus (Table 2). The timing of systemic symptoms (Table 2) and the CP expression levels (Fig. 7A) were similar for the two mutants (p25ACG and $\mathrm{p} 22.5 \mathrm{ACG}$ ) and the wild-type virus. RT-PCR and DNA sequencing confirmed that the mutated sites were retained in the progeny viruses in systemically infected leaves.

Two double-site mutants, p27CAG+p22.5ACG and p25ACG+p22.5ACG (Fig. 5), were constructed to further confirm the function of p27 and p25. On both local and systemically infected leaves, mutants p27CAG+p22.5ACG and p27CAG shared similar mild symptoms (Fig. 6C and F). By contrast, mutants p25ACG+p22.5ACG and p25ACG elicited symptoms similar to those of the wild-type virus (Fig. 6B and E). Levels of virus replication in transfected kenaf protoplasts (Fig. 8B) and CP expression in systemically infected leaves at 28 dpi were comparable among all the mutants (Fig. 7A). Based on these results, we conclude that p27 affects symptom expression, that p25 may be involved in the onset of systemic symptom and facilitation of virus long distance movement, and that p22.5 appears not to function in symptom development of HCRSV.
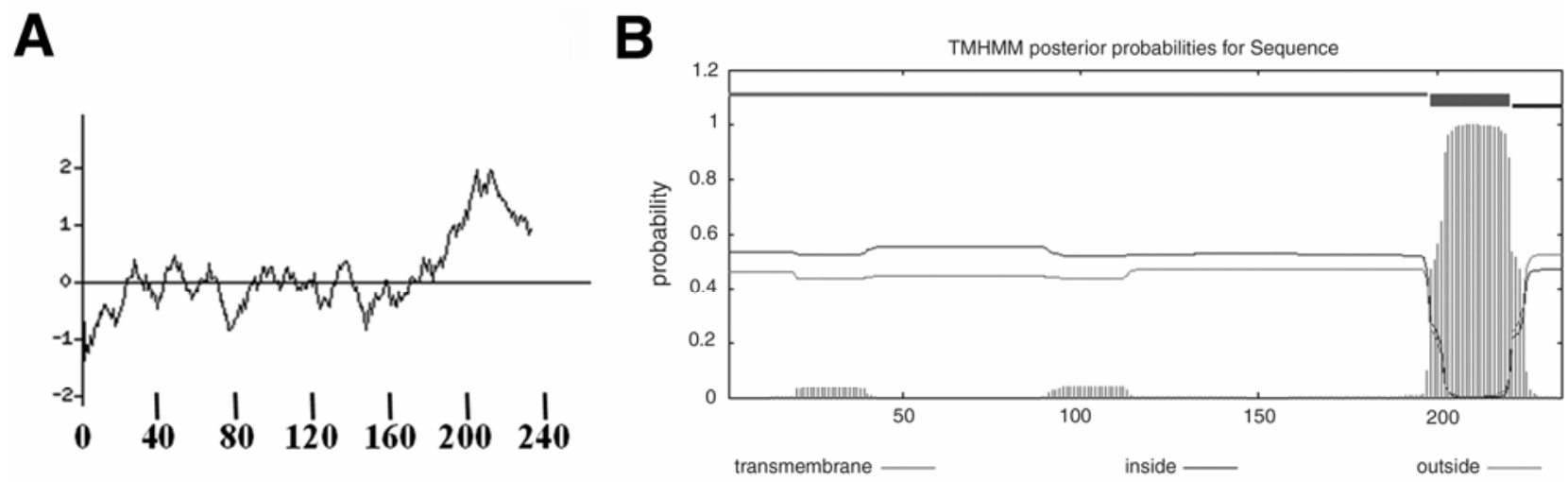

Fig. 3. Prediction of the hydrophobicity and transmembrane domain of Hibiscus chlorotic ringspot virus (HCRSV) p27. A, Kyte-Doolittle x-1 method of calculating hydrophilicity over a window length of 30, hydropathy plots showed that HCRSV p27 has a highly hydrophobic domain at its C-terminal (amino acids 198 to 220). B, The TMHMM 2.0 software predicted a transmembrane domain (amino acids 198 to 220) and its N-terminus (amino acids 1 to 197) which is located outside the membrane. The C-terminus (amino acids 221 to 235) is located in the cytoplasm. 


\begin{tabular}{|l|l|}
\hline GFP \\
NOS \\
\hline
\end{tabular}

p27-GFP

\begin{tabular}{|l|l|l|l|l|}
\hline $35 \mathrm{~S}$ & 198 & 220 \\
\hline & $\mathbf{p 2 7}$ & GFP & NOS \\
\hline
\end{tabular}

\begin{tabular}{|c|c|c|}
\hline $35 \mathrm{~S}$ & 197 \\
\hline $\mathbf{p 2 7 N 1 9 7}$ & GFP \\
\hline
\end{tabular}

\section{p38-GFP}
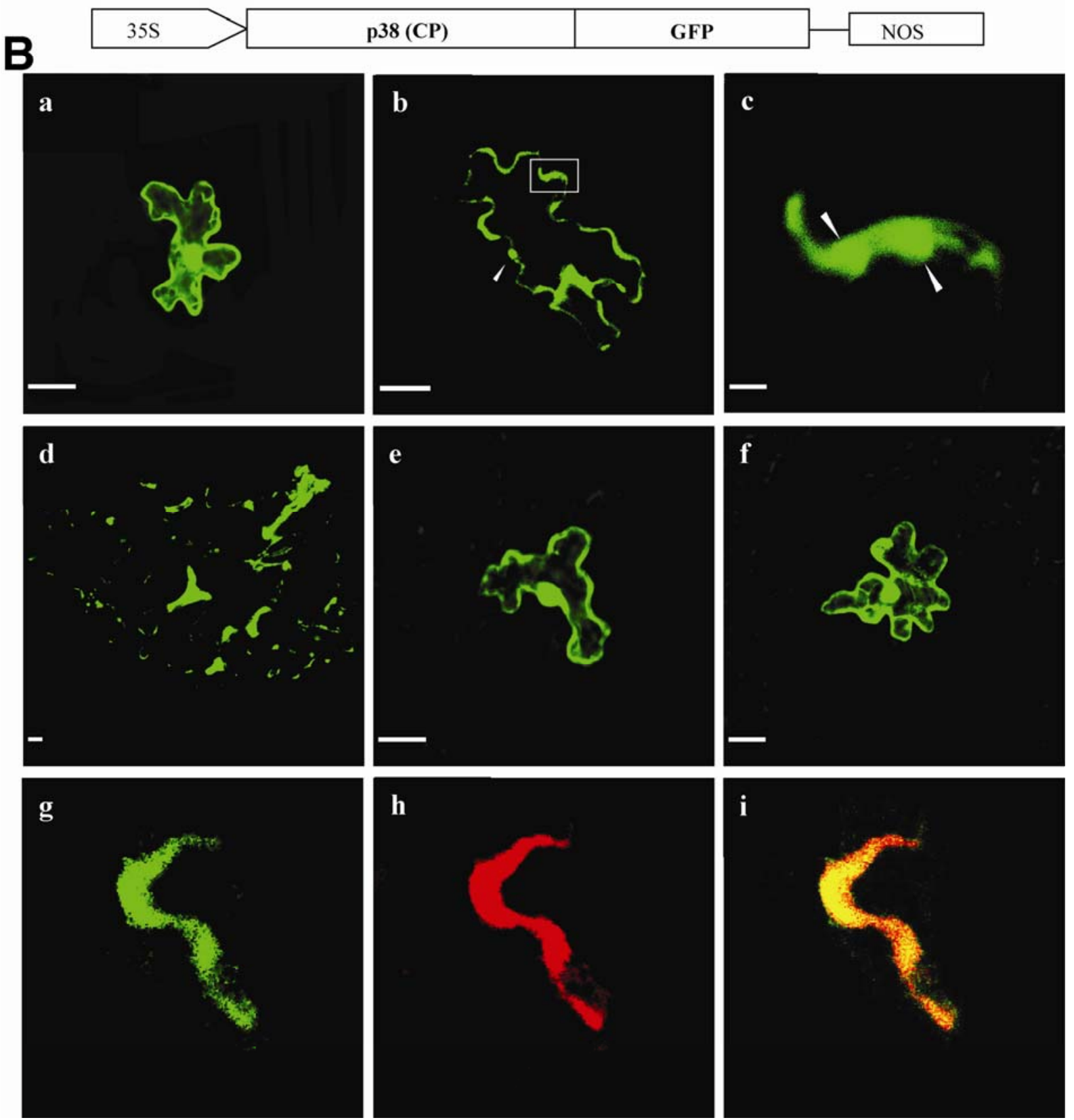

Fig. 4. Subcellular localization of p27-GFP (green fluorescent protein) fusion protein in kenaf epidermal cells. A, Schematic representation of constructs used for agroinfiltration. Boxes indicate coding regions. 35S indicates the Cauliflower mosaic virus 35S RNA promoter and NOS represents the nos terminator. The solid vertical bar in the construct p27-GFP represents the transmembrane domain in p27. The construct p27N197-GFP expresses the Nterminal 197 amino acids but not the deleted C-terminal amino acids from 198 to 235. B, Localization of free GFP and GFP fusion proteins. (a) Fluorescence of free GFP localizes in both nucleus and cytoplasm of a single cell. (b) p27-GFP fusion protein localizes to membrane structures close to the cell walls (arrowheads). (c) Close-up image of the membrane structures (arrowheads) as shown in b. (d) A cluster of cells showing green fluorescence from p27-GFP fusion protein. (e) Fluorescence in a single cell from p27N197-GFP and (f) p38-GFP, showing green fluorescence in the nucleus and cytoplasm of a single cell. Bar $=20 \mu \mathrm{m}$, except for $\mathrm{c}=2 \mu \mathrm{m}$. (g) through (i), The p27-GFP fusion protein was localized to membrane structures. Using a membrane-specific dye, FM4-64 (red in h), the merged photo in i confirms the p27-GFP fusion protein was localized to membrane structures. 
To investigate whether the delayed systemic symptoms were caused by the retardation of virus movement, a timecourse analysis of $\mathrm{CP}$ accumulation in the systemically infected leaves was carried out (8 to $21 \mathrm{dpi}$ ) daily from the onset of systemic symptoms of wild-type infection to the appearance of systemic symptoms of mutants p25ACG and p25ACG+p22.5ACG infection. Total proteins from $40 \mathrm{mg}$ of leaves from each sample were probed with anti-HCRSV CP antiserum. The results (Fig. 7C) indicate that the delayed systemic symptom phenotype corresponds to the delayed virus movement. Therefore, we conclude that p25 potentiates effective virus spread in HCRSVinfected kenaf.

\section{The absence of p27 and p25 abolishes} symptom expression and cell-to-cell movement.

To further investigate the involvement of p27 and p25 in the virulence of HCRSV on kenaf plants, four mutants representing p25trunc, p22.5trunc, p27CAG+p25ACG, and p27CCG+p25ACG+p22.5ACG (Fig. 5), which do not express p27 or p25, were tested. No signs of local infection or systemic symptoms were observed during the 30-day assay period (Fig. 6A and D), and no RT-PCR product was detected from the inoculated leaves with any of the four constructs (Fig. 8A).

The levels of CP expression (Fig. 7B), gRNA, and sgRNAs (Fig. 8B) of the four mutants were comparable to that of the wild type in transfected kenaf protoplasts. However, neither viral RNA nor CP was detected in inoculated or upper leaves when the four mutants were inoculated onto whole plants. These results showed that absence of both p27 and p25 resulted in a lack of both symptom expression and virus movement in kenaf plants.

\section{DISCUSSION}

In this study, a new protein, p27, was detected in HCRSVtransfected kenaf protoplasts through fusion with a Flag tag. The p27 ORF of HCRSV has a CUG start codon, and it has the potential to encode two other isoforms, p25 and p22.5. Although the $5^{\prime}$ terminus of ORF p27 is nested within the $3^{\prime}$ region of ORF p9, the p25 and p22.5 ORF are nested further downstream within the $\mathrm{CP}$ gene. These features are also found in two Taiwanese isolates of HCRSV (Y. C. Chang, personal communication). For all three isoforms, no homologous protein was found in the GenBank database. Among the carmoviruses, only Cowpea mottle virus $(\mathrm{CPMoV})$ possesses a similar p28 ORF (You et al. 1995) at the corresponding genomic position. However, the p28 ORF does not encode any isoforms and its existence has not yet been demonstrated in vivo. Amino-acid sequence alignment of the CPMoV p28 with the HCRSV p27 revealed only $11.5 \%$ homology.

Table 1. Localization of green fluorescent protein (GFP) and p27-GFP fusion protein in agroinfiltrated epidermal cells of kenaf leaves

\begin{tabular}{lllcc}
\hline & \multicolumn{4}{c}{ Green fluorescence loci in cluster cells $^{\mathbf{a}}$} \\
\cline { 2 - 5 } Time (h) & \multicolumn{1}{c}{ GFP } & p27-GFP $^{\mathbf{b}}$ & p27N197-GFP & p38-GFP \\
\hline 48 & $6.7(7 / 104)$ & $54.0(27 / 50)$ & $10.0(12 / 120)$ & $13.4(15 / 112)$ \\
84 & $6.4(8 / 124)$ & $60.7(74 / 122)$ & $8.0(10 / 125)$ & $11.5(14 / 122)$ \\
120 & $9.5(12 / 127)$ & $65.8(73 / 111)$ & $7.1(8 / 113)$ & $12.3(13 / 106)$ \\
\hline
\end{tabular}

${ }^{a}$ Percentage of fluorescent cell clusters observed in agroinfiltrated epidermal leaf cells of kenaf, with constructs expressing GFP, p27-GFP, p27N197-GFP, and p38-GFP fusion proteins, respectively. Fluorescent cell clusters are defined as two or more adjacent cells that emit green fluorescence. The total number of fluorescent cell clusters relative to total number of sites showing green fluorescence is shown in parentheses.

${ }^{\mathrm{b}}$ Strong green fluorescence was observed in agroinfiltrated leaves with the expression of p27-GFP fusion protein after $72 \mathrm{~h}$. Relatively few numbers of cells showed green fluorescence at the earlier sampling at $48 \mathrm{~h}$.
Immunodetection using anti-FLAG M2 mAb showed that p27 was expressed at a low level but had a much stronger signal in transfected protoplasts when the ${ }^{2570} \mathrm{CUG}$ start codon was changed to ${ }^{2570} \mathrm{AUG}$ (Fig. 2C). Non-AUG initiated translation has been shown to be a strategy of gene expression among plant viruses and these products play certain specific roles (Drugeon et al. 1999; Fütterer et al. 1996). The 25-kDa protein of Soilborne wheat mosaic virus is initiated at a CUG codon and has a downstream in-frame AUG initiating a 19-kDa protein (Shirako 1998). That is similar to the situation here, in which the HCRSV p27 is expressed through a CUG codon and produces an in-frame p25 through an AUG codon. Low-level expression of p27 seems to be sufficient to influence symptom severity. When both p27 and p25 were abolished, infection could not be detected on either inoculated or uninoculated kenaf leaves. Although p25 has yet to be detected in vivo, we speculate that it is expressed during infection, because the ORF has a role in symptom expression and virus movement. Similarly, although there is no evidence for the function of p22.5 and it is not expressed to detectable levels in infected kenaf, we are unable to rule out its existence or its possible interactions with the host.

The translational strategy of p27 is intricate. Our experimental results showed that when the noncanonical start codon ${ }^{2570} \mathrm{CUG}$ was changed to ${ }^{2570} \mathrm{AUG}$, there was no detectable expression of the CP. Both CP and p27 are translated from sgRNA2 (D. C. Y. Koh, unpublished data) but in different reading frames. The most probable mechanism of regulating the translation of the two proteins is by leaky scanning, which has been shown to produce two separately initiated proteins in eukaryotic and viral mRNAs (Kozak 2002). Most recently, Omarov and associates (2005) reported that the CP gene of Satellite panicum mosaic virus directs two proteins by translation from the first (AUG1) and the third (AUG3) start codons, both of which are in the same translational frame and have different biochemical characteristics.

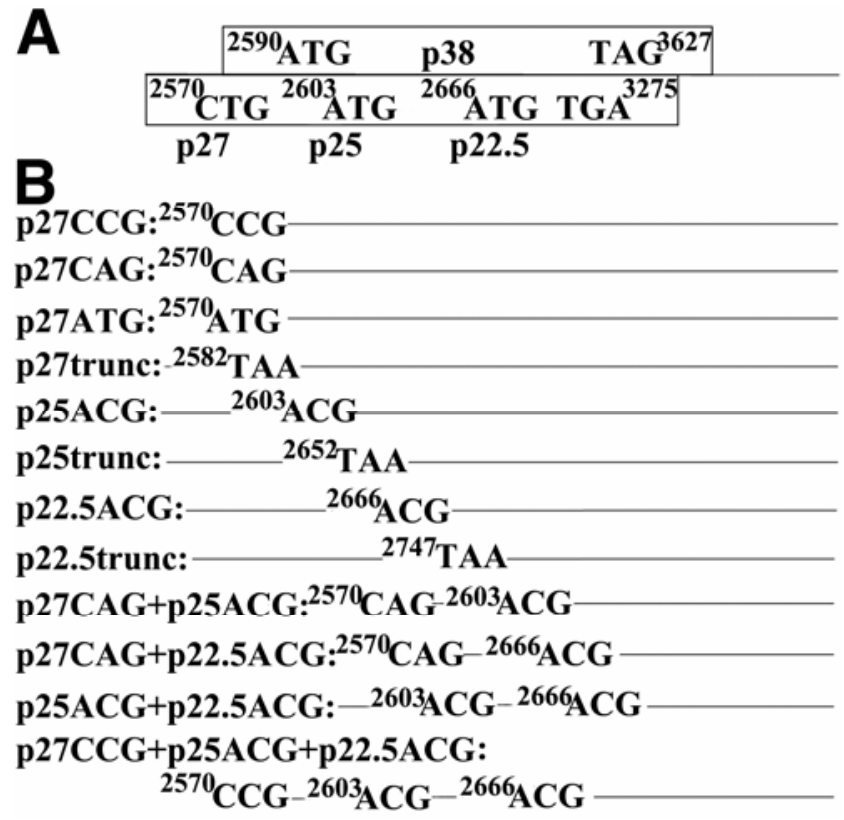

Fig. 5. Schematic representation (not drawn to scale) of open reading frames (ORF) p38 and p27 and mutants used in infection assay. A, Rectangles represent the ORF p38 and p27. The start codons of the p27 isoforms, which are CUG for p27 and AUG for both p25 and p22.5. B, Mutants are named after the location of mutated nucleotides. Construct p27CCG+ p25ACG+p22.5ACG indicates that only p38 but not p27, p25, or p22.5 is expressed. Except for construct p27ATG, which changes one amino-acid residue of $\mathrm{p} 9$, the other constructs do not alter codons of p9 or p38 (coat proteins). 
Based on structure predictions, p27 and its isoforms are highly hydrophobic and have a transmembrane domain at the carboxyl termini (Fig. 3), which suggests that they are membrane-associated proteins. Subcellular localization of p27-GFP fusion protein confirmed that HCRSV p27 is a membrane protein (Fig. 4B). Membrane proteins encoded by plant viruses are important to the virus life cycle and have been found to be required for virus replication, movement, or both. For example, the 6-kDa protein of Tobacco etch virus targets replication complexes to membranous sites of RNA synthesis (Schaad et al. 1997), and the hydrophobic segment of Potato virus $X$ (PVX) TGBp3 is necessary for intracellular trafficking and virus cellto-cell movement (Krishnamurthy et al. 2003; Schepetilnikov et al. 2005).
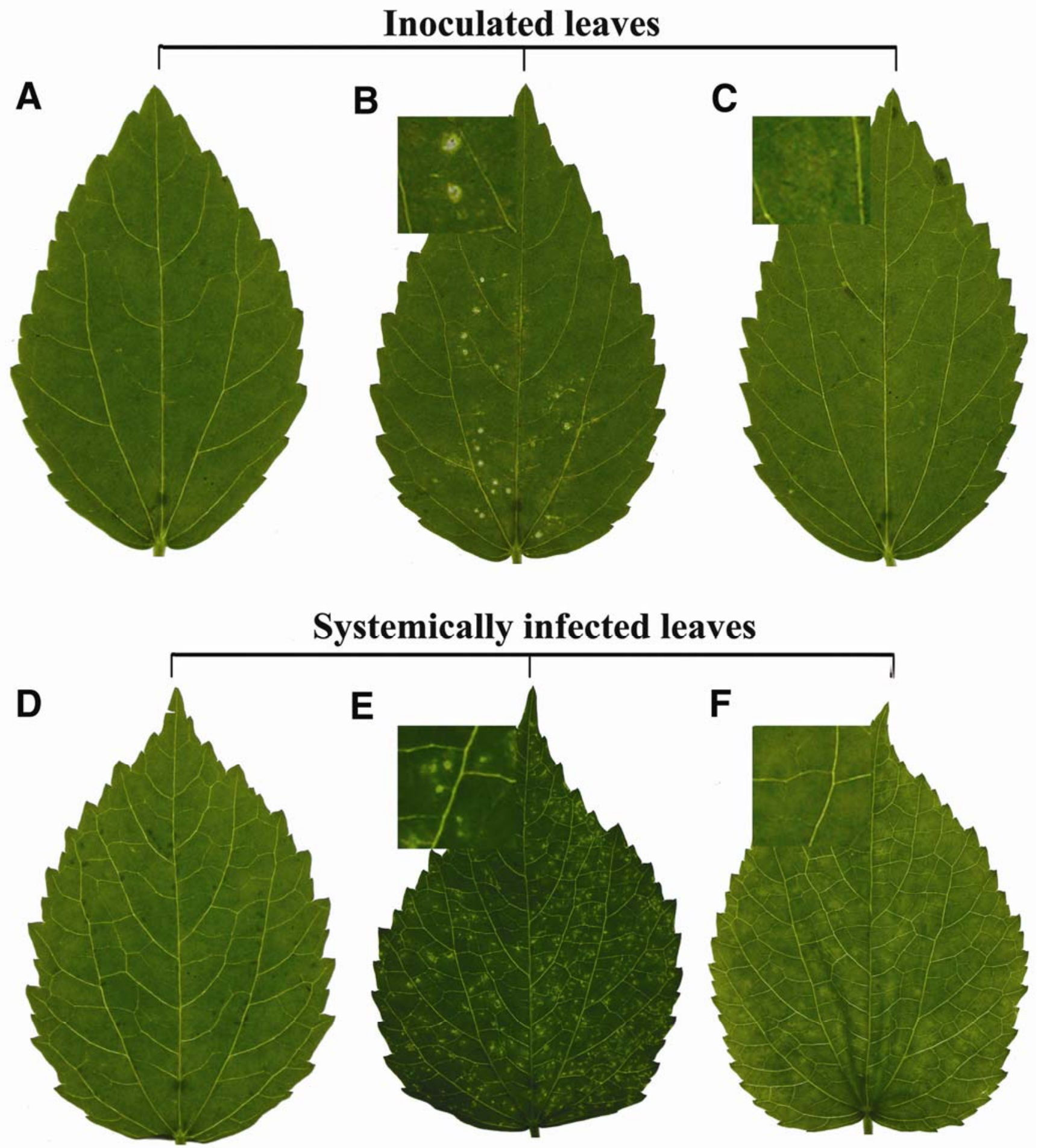

Fig. 6. Representative phenotypes of Hibiscus chlorotic ringspot virus p27 and its isoforms on inoculated and systemically infected kenaf leaves that were harvested at 6 and 30 days after inoculation, respectively. A and D, Representative leaves that lack symptom expression after inoculation with mutants p27ATG, p25trunc, p22.5trunc, p27CAG+p25ACG, p27CCG+p25ACG+p22.5ACG, and buffer only. B and E, Representative leaves showing local lesion on the inoculated leaf and chlorotic ringspots on systemically infected leaves after inoculation with the wild-type virus (p223) and mutants p25ACG, p22.5ACG, and p25ACG+p22.5ACG. C and F, Representative leaves infected by mutants p27CCG, p27CAG, p27trunc, and p27CAG+p22.5ACG, which induce milder symptoms. 
Due to the relative positions of $\mathrm{p} 9, \mathrm{CP}, \mathrm{p} 27$, and its isoforms in the genome, mutations could only be made on the three start codons or by introducing stop codons downstream to truncate p27 and its isoforms without affecting the other ORF. Because all of the mutants could replicate equally well in transfected kenaf protoplasts (Fig. 8B), it is clear that p27 and its isoforms are not required for virus replication.

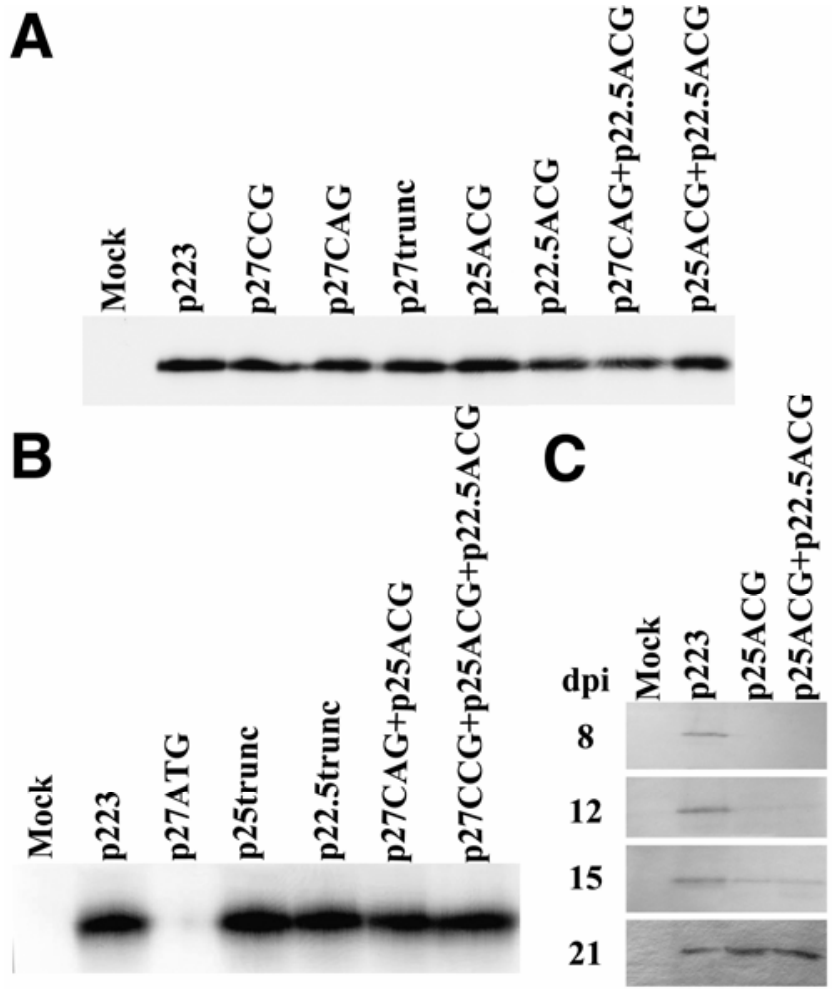

Fig. 7. Western blot analysis of Hibiscus chlorotic ringspot virus (HCRSV) coat protein (CP) (38 kDa) expression in vivo. A, Total proteins extracted from systemically infected kenaf leaves at 28 days postinoculation (dpi). B, Accumulation of CP in transfected kenaf protoplasts at $72 \mathrm{~h}$ posttransfection. C, Timecourse analysis of CP accumulation in systemically infected leaves on $8,12,15$, and 21 dpi with buffer (Mock), wild-type (p223), and two mutants (p25ACG and p25ACG+ $\mathrm{p} 22.5 \mathrm{ACG})$ that induced delayed systemic symptoms. Total proteins from $40 \mathrm{mg}$ of leaves or $1 \times 10^{5}$ protoplasts of each sample were probed with anti-HCRSV CP antiserum. Mock represents mock inoculation or mock transfection.
The p27CCG, p27CAG, and p27trunc mutants were unable to express p27 and elicited attenuated symptoms. This shows that p27 is a determinant for enhancement of HCRSV symptom severity in kenaf. However, overexpression of p27 with the ${ }^{2570} \mathrm{CUG}$ to ${ }^{2570} \mathrm{AUG}$ mutation failed to develop systemic symp-

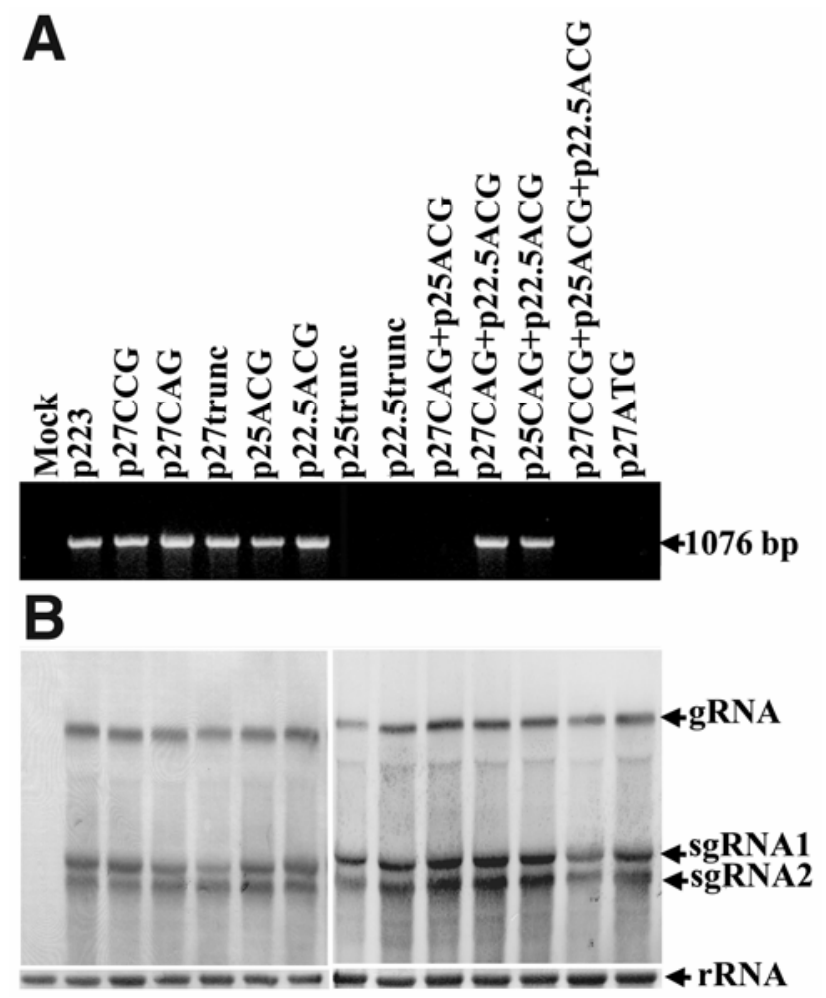

Fig. 8. Analyses of viral RNA replication in inoculated leaves and transfected kenaf protoplasts. A, Detection of replication of Hibiscus chlorotic ringspot virus (HCRSV) p27 isoform mutants on inoculated kenaf leaves by reverse transcription-polymerase chain reaction. Total RNA was extracted from the inoculated leaves. The arrow indicates the expected product of 1,076 bp, encompassing nucleotides (nt) 2, 200 to 3,275 of the viral genome. B, Northern blot analysis of total RNA from kenaf protoplasts transfected with p223 and mutant transcripts after $24 \mathrm{~h}$ incubation. Total RNA $(5 \mu \mathrm{g})$ was loaded in each lane and was probed with digoxigeninlabeled RNA probe complementary to the HCRSV genome at nt 3,068 to 3,911. Mock represents mock inoculation. HCRSV genomic RNA (gRNA), subgenomic RNAs (sgRNA), and kenaf (28S) rRNA are indicated. The band intensity of rRNA indicates the relative amount of samples loaded.

Table 2. Comparison of symptom appearance and severity for mutants of p27 and its isoforms on inoculated and systemically infected upper kenaf leaves

\begin{tabular}{|c|c|c|c|c|}
\hline \multirow[b]{2}{*}{ Constructs } & \multirow[b]{2}{*}{ Number of plants ${ }^{a}$} & \multicolumn{2}{|c|}{ Symptom appearance $(\text { dpi })^{b}$} & \multirow[b]{2}{*}{ Symptom severity ${ }^{c}$} \\
\hline & & Inoculated & Systemically infected & \\
\hline Mock & $0 / 12$ & - & - & - \\
\hline p223 (wild type) & $12 / 12$ & 4 to 6 & 8 to 12 & +++ \\
\hline p27CCG & $45 / 45$ & 4 to 6 & 8 to 12 & ++ \\
\hline p27CAG & $45 / 45$ & 4 to 6 & 8 to 12 & ++ \\
\hline p27ATG & $0 / 42$ & - & - & - \\
\hline p25ACG & $42 / 42$ & 4 to 6 & 16 to 20 & +++ \\
\hline p22.5ACG & $42 / 42$ & 4 to 6 & 8 to 12 & +++ \\
\hline p27trunc & $45 / 45$ & 4 to 6 & 8 to 12 & ++ \\
\hline p25trunc & $0 / 42$ & - & - & - \\
\hline p22.5trunc & $0 / 42$ & - & - & - \\
\hline p27CAG+p25ACG & $0 / 24$ & - & - & - \\
\hline $\mathrm{p} 27 \mathrm{CAG}+\mathrm{p} 22.5 \mathrm{ACG}$ & $24 / 24$ & 4 to 6 & 8 to 12 & ++ \\
\hline $\mathrm{p} 25 \mathrm{ACG}+\mathrm{p} 22.5 \mathrm{ACG}$ & $24 / 24$ & 4 to 6 & 16 to 20 & +++ \\
\hline $\mathrm{p} 27 \mathrm{CCG}+25 \mathrm{ACG}+\mathrm{p} 22.5 \mathrm{ACG}$ & $0 / 42$ & - & - & - \\
\hline
\end{tabular}

${ }^{\text {a }}$ Plants showing symptoms/total number of plants tested. Results are from three independent experiments.

${ }^{\mathrm{b}}$ Time required before appearance of local lesions on inoculated leaves and symptoms on systemically infected upper leaves in days postinoculation (dpi).

${ }^{\mathrm{c}}$ Severity of symptoms on systemically infected upper leaves. +++ = extensive spread of necrotic spots (equivalent to wild type); ++ = mild mosaic and veinbanding or mottling; and $-=$ no symptoms. 
toms and viral RNA could not be detected in the inoculated leaves. Since the mutant p27ATG has one amino-acid residue of $\mathrm{p} 9$ altered $\left({ }^{76} \mathrm{Thr}\right.$ to $\left.{ }^{76} \mathrm{Asn}\right)$, it might have affected cell-tocell movement of the virus. In addition, there was no detectable CP in transfected protoplasts, but precise analyses of the roles of HCRSV $\mathrm{p} 9$ and $\mathrm{CP}$ in virus movement have not been evaluated. For TCV, p9 is required for cell-to-cell movement while CP is host-dependent for cell-to-cell movement (Hacker et al. 1992; Heaton et al. 1991; Li et al. 1998), so it is possible that both of these genes have functions in either local or long distance movement, of both, of HCRSV.

For some plant viruses, CP participates in replication (Asurmendi et al. 2003; Neeleman et al. 1993; van der Kuyl et al. 1991) and symptom expression (Banerjee et al. 1995; Heaton et al. 1991, 1995; Neeleman et al. 1991; Rao and Grantham 1995; Shintaku et al. 1992; Suzuki et al. 1995). Our work shows that the HCRSV CP is not involved in replication. Recently, the HCRSV CP but not p27 has been shown to be a suppressor of post-transcriptional gene silencing (PTGS) (Meng et al. 2006). There may also be additional virus-host interactions involving $\mathrm{p} 27$, the $\mathrm{CP}$, and one or more unidentified host factors that regulate p27 and the CP during HCRSV infection.

The absence of p25 delayed virus systemic movement and development of systemic symptoms. However, p25 is not a suppressor of PTGS (X. Z. Liang, unpublished data). Similarly, the cysteine-rich protein of Potato mop-top virus (Lukhovitskaya et al. 2005) and the $4.8-\mathrm{kDa}$ protein of Tobacco mosaic virus (TMV) (Canto et al. 2004) have been reported to enhance virulence, but they are not PTGS suppressors, either. Unfortunately, we were unable to detect the p 25 protein in transfected kenaf protoplasts, although the total proteins were concentrated or fractionated and enriched by differential centrifugation.

Because both p27 and p25 possess the common $\mathrm{C}$ terminus and the transmembrane domain, it is predicted that p25 is also expected to be membrane-bound and have the same subcellular localization as p27. The membrane localization suggests that $\mathrm{p} 27$, p25, or both might be involved in virus movement.
The clustered cell fluorescence of p27-GFP fusion protein showed that it may participate in cell-to-cell movement in kenaf epidermal cells (Table 1). Similar results with the movement proteins of TMV, PVX, and Cucumber mosaic virus indicated that intracellular virus movement requires membrane association (Tamai and Meshi 2001a and b). Considering that p25 may potentiate virus movement, our hypothesis is that $\mathrm{p} 25$ possesses the ability to facilitate the increase of the plasmodesmatal size exclusion limit and thus promote virus movement.

In order to explore the interactions of p27 and its isoforms with the host factors, further studies using protein-protein interaction identification systems and microarray techniques will be necessary to elucidate the mechanisms whereby p27 and its isoforms modulate symptom severity and systemic movement of HCRSV.

\section{MATERIALS AND METHODS}

\section{Prediction of the hydrophobicity and transmembrane domains of p27.}

Hydropathy plot (Weizmann Institute of Science Bioinformatics and Biological Computing website) and TMHMM version 2.0 (Krogh et al. 2001) programs were used to predict the hydropathic profile and transmembrane helices of $\mathrm{p} 27$, respectively.

\section{Construction of p27 and isoform mutants.}

All mutants were derived from p223, which is the fulllength biologically active cDNA clone of HCRSV (Huang et al. 2000). Mutations were confirmed by restriction enzyme digestions and automated DNA sequencing. Oligonucleotide primers used for cloning and the modified clones are summarized in Table 3.

To generate flag-tagged p27 for in vivo immunodetection, p27 ${ }_{N}$ Flag and p27atgFlag constructs (Fig. 2A) were prepared, using the forward primers 2570CUGflag-f, and 2570AUGflag$\mathrm{f}$, respectively with a common reverse primer Rev-SacII. The p27 $\mathrm{C}$ Flag construct was made through overlapping PCR with

Table 3. Oligonucleotides used for cloning and mutagenesis

\begin{tabular}{|c|c|c|}
\hline Primers $^{\mathrm{a}}$ & Nucleotide position $^{b}$ & Sequences $\left(5^{\prime} \text { to } 3^{\prime}\right)^{\mathrm{c}}$ \\
\hline 2570CUGflag-f & 2,558 to 2,586 & TAGTAGTTAACACTGGACTACAAGGACGACGATGACAAAGAAAGTAACCAACC \\
\hline 2570AUGflag-f & 2,558 to 2,586 & TAGTAGTTAACAATGGACTACAAGGACGACGATGACAAAGAAAGTAACCAACC \\
\hline 3275Flag-r & 3,257 to 3,279 & AATCATTTGTCATCGTCGTCCTTGTAGTCCATCCACATCCCCTTCAG \\
\hline 3275Flag-f & 3,272 to 3,292 & ATGGACTACAAGGACGACGATGACAAATGATTGGAGAGCTGTTCC \\
\hline Rev-SacII & 3,888 to 3,911 & agttceccgcggcceGGGCTGCCTCACAACTACTATGGC \\
\hline 2570CCG-f & 2,558 to 2,586 & TAGTAGTTAACACCGGAAAGTAACCAACC \\
\hline 2570CAG-f & 2,558 to 2,586 & TAGTAGTTAACACAGGAAAGTAACCAACC \\
\hline 2570ATG-f & 2,558 to 2,586 & TAGTAGTTAACAATGGAAAGTAACCAACC \\
\hline 2603ACG-r & 2,587 to 2,617 & AACGGCTGGGTCGTTCTTCTGCAGCATTGTGG \\
\hline 2603ACG-f & 2,598 to 2,621 & GAAGAACGACCCAGCCGTTCAACG \\
\hline 2582TAA-f & 2,558 to 2,593 & TAGTAGTTAACACTGGAAAGTAACTAACCAACCACAATGC \\
\hline 2666ACG-r & 2,652 to 2,675 & GCCCACCCGTCGTTCTTAAGTTTG \\
\hline 2666ACG-f & 2,654 to 2,680 & AACTTAAGAACGACGGGTGGGCTGCTC \\
\hline 2652TAA-r & 2,641 to 2,665 & CGTTCTTAAGTTTTATTGCCCAAGG \\
\hline 2652TAA-f & 2,641 to 2,665 & CCTTGGGCAATAAAACTTAAGAACG \\
\hline 2748TAA-r & 2,731 to 2,752 & GCTTTTTTGAGAAGTAACAGG \\
\hline 2748TAA-f & 2,737 to 2,766 & АCTTCTCAAATAAAGCTGTCAACACTTAAG \\
\hline 2570CUG-f & 2,558 to 2,586 & TAGTAGTTAACACTGGAAAGTAACCAACC \\
\hline p27SacI-f & 2,569 to 2,586 & agagagctcgCTGGAAAGTAACCAACC \\
\hline p27BamHI-r & 3,258 to 3,274 & taaggatcc CATCCACATCCCCTTC \\
\hline p27N197BamHI-r & 3,138 to 3,160 & taaggatccGTATTTGCAGCATGGCACTATCC \\
\hline p38XmaI-f & 2,588 to 2,607 & atacccggggaATGCTGCAGAAGAATGAC \\
\hline p38NcoI-r & 3,606 to 3,624 & tatccatggAGTTCCTACAGGCCCACC \\
\hline
\end{tabular}

a ' $\mathrm{f}$ ' and ' $\mathrm{r}$ ' indicate that the primer corresponds to Hibiscus chlorotic ringspot virus (HCRSV) genome RNA or is complementary to genome RNA, respectively.

${ }^{\mathrm{b}}$ Positions of the primers in complete sequence of Hibiscus chlorotic ringspot virus (HCRSV) genome are shown.

${ }^{c}$ Oligonucleotide sequences are shown in 5' to $3^{\prime}$ direction. The HpaI site is shown in italics. Mutations in HCRSV sequence are indicated in bold. The sequences coding FLAG epitope are underlined. The lower case is the added non-HCRSV sequence, and the corresponding restriction enzyme site in the primer name is in italics. 
two pairs of primers, 2570CUG-f and 3275Flag-r and 3275Flag-f and Rev-SacII. The PCR fragments were ligated into linearized p223 that had been digested with $\mathrm{HpaI} / \mathrm{SacII}$.

The constructs for infectivity assays are represented in Figure 5. Mutants designated p27CCG, p27CAG, and p27ATG, which altered the ${ }^{2570} \mathrm{CUG}$ start codon in p223, were generated using the forward primers 2570CCG-f, 2570CAG-f, and 2570ATG-f, respectively, with a common reverse primer RevSacII. The PCR fragments were digested separately with $H p a \mathrm{I} / \mathrm{SacII}$ and were ligated into the corresponding sites in p223. The p27CCG and p27CAG mutations did not affect the amino-acid residues of the p9, whereas p27ATG altered the ${ }^{76} \mathrm{Thr}$ of $\mathrm{p} 9$ to ${ }^{76} \mathrm{Asn}$. Similarly, the p25ACG construct that changed the ${ }^{2603}$ AUG start codon of p25 used the primers 2603ACG-f and Rev-SacII. Mutant p27trunc changed the ${ }^{2582} \mathrm{CAA}$ to ${ }^{2582} \mathrm{UAA}$, to generate a premature termination of p27, just after the four N-terminal amino acids. This mutant was constructed using the primers 2582TAA-f and Rev-SacII. The p25ACG and p27trunc constructs did not affect the amino-acid residues of the two overlapping ORF, p9 and CP.

Overlapping PCR was used to obtain the p22.5ACG, p25trunc, and p22.5trunc mutants. To construct the p22.5ACG plasmid, two PCR fragments (I and II) were amplified with two pairs of primers, 2570CUG-f and 2603ACG-r and 2603ACG-f and Rev-SacII, respectively. The resulting fragments were gel-purified and mixed with equal molar amounts of templates for the second-round PCR, using primers 2570CUG-f and Rev-SacII. For p25trunc plasmid construction, two pairs of primers, 2570CUG-f and 2652TAA-r and 2652TAA-f and Rev-SacII, were used. After HpaI and SacII digestion, the PCR fragments were separately ligated into p223. The three mutants did not alter the coding sequence of the overlapping $\mathrm{CP}$.

To construct p27CAG+p25ACG and p27CAG+p22.5ACG, the 2570CAG-f and Rev-SacII PCR primers were used with $\mathrm{p} 25 \mathrm{ACG}$ or $\mathrm{p} 22.5 \mathrm{ACG}$ as the templates. To generate p25ACG+p22.5ACG, overlapping PCR was carried out with two template fragments, p25ACG and p22.5ACG (fragments I and II, respectively), using the primers 2603ACG-f and RevSacII. The p27CCG+p25ACG+p22.5ACG mutants were designed using the primers 2570CCG-f and Rev-SacII, with p25ACG+p22.5ACG as template.

\section{In vitro transcription and inoculation.}

Appropriate plasmid DNA constructs were linearized with SmaI, and in vitro RNA transcripts were synthesized using T7 RNA polymerase. A $1: 1$ mixture of the transcripts $(0.5 \mu \mathrm{g}$ RNA per leaf) and GKP (50 mM glycine, $30 \mathrm{mM} \mathrm{K}_{2} \mathrm{HPO}_{4}$, $\mathrm{pH}$ 9.2, $1 \%$ bentonite, $1 \%$ celite) buffer (Petty et al. 1989) was inoculated onto Carborundum-dusted leaves of 3- to 4-weekold kenaf plants. The plants were maintained at 28 to $30^{\circ} \mathrm{C}$ in a plant-growth room. Kenaf protoplasts $\left(4 \times 10^{5}\right)$ were prepared and transfected with $10 \mu \mathrm{g}$ of RNA as described earlier (Liang et al. 2002a).

\section{Western and Northern blots.}

Total proteins were extracted from kenaf leaves or protoplasts at $72 \mathrm{hpt}$, according to Liang and associates (2002b). Protein samples were separated by $12 \%$ sodium dodecyl sulfate-polyacrylamide gel electrophoresis (Laemmli 1970) and were analyzed by Western blots, probed with a rabbit antiserum against a bacterial-expressed HCRSV CP or anti-FLAG M2 mAb.

Total RNA was isolated from transfected protoplasts at 24 hpt and was analyzed by Northern blots. Accumulation of both the viral gRNA and sgRNAs was determined using a digoxigenin-labeled antisense RNA probe complementary to nt 3,068 to 3,911 of HCRSV RNA.
Agrobacterium tumefaciens-mediated transient expression and confocal laser scanning microscopy.

For construction of GFP fusion proteins, pCam35S-GFP (provided by G. Zejian, China Agricultural University, Beijing) was used. The coding regions of HCRSV p27 and CP (p38) ORF were amplified from p223, using the primers p27SacI-f and p27BamHI-r and p38XmaI-f and p38NcoI-r, respectively. The PCR fragments were digested with SacI and BamHI and $X m a I$ and NcoI separately and were ligated into the corresponding sites in pCam35S-GFP.

To investigate the specificity and localization of $\mathrm{p} 27$-GFP fusion protein targeting subcellular components, the transmembrane domain deletion mutant pHCRSVp27N197-GFP was made using primers p27SacI-f and p27N197BamHI-r, and the PCR product was ligated into pCam35S-GFP. All vector constructs are depicted in Figure 4A.

For transient expression, 3- to 4-week-old kenaf leaves were used. Agrobacterium tumefaciens EHA105 suspensions were prepared as described earlier (Yang et al. 2000). Agrobacteriummediated transient transformation was conducted on young fully expanded leaves. The bacterial suspension was infiltrated into intercellular spaces of leaves using a 1-ml sterile syringe without a needle. About $100 \mu \mathrm{l}$ of bacterial suspension was infiltrated into each site. On a single kenaf leaf, 6 to 10 sites, separated by veins, were infiltrated. After agroinfiltration, the kenaf plants were incubated at 28 to $30^{\circ} \mathrm{C}$ in a plant-growth room.

To observe green fluorescence in the infiltrated leaves, leaf disks encompassing the infiltrated sites were excised, were mounted as live tissue on microscope slides, and were observed immediately. For cellular localization of p27-GFP, a membranespecific dye FM4-64 was used to stain the sample. A confocal laser scanning microscope (Olympus FLOWVIEW300) was used to obtain images.

\section{ACKNOWLEDGMENTS}

We thank A. O. Jackson, the University of California, Berkeley, P. Palukaitis, Scottish Crop Research Institute, and M. Zaitlin, Cornell University, for editing this manuscript and Y. C. Chang from National Taiwan University for sharing the unpublished sequence information of HCRSVTaiwan isolates. We also thank R. Hull from Norwich, U.K. for helpful discussions, Z. J. Guo from the China Agricultural University for plasmid pCam35S-GFP, N. I. Navqi from Temasek Life Sciences Laboratory for membrane-specific dye FM4-64, G. L. Loy and J. Yang for their help in operating the confocal laser scanning microscope and collecting data in the Department of Biological Sciences, National University of Singapore (NUS). This work was supported by the National Natural Science Foundation of China (number 30340074), the State Key Laboratory of Agrobiotechnology, Beijing, China, the NUS International Collaboration Fund, and research grants R-154-000-189-112 and R-154-000-252-112.

\section{LITERATURE CITED}

Asurmendi, S., Berg, R. H., Koo, J. C., and Beachy, R. N. 2003. Coat protein regulates formation of replication complexes during tobacco mosaic virus infection. Proc. Natl. Acad. Sci. U.S.A. 101:1415-1420.

Banerjee, N., Wang, J. Y., and Zaitlin, M. 1995. A single nucleotide change in the coat protein gene of tobacco mosaic virus is involved in the induction of severe chlorosis. Virology 207:234-239.

Brunt, A. A., Crabtree, K., Dallwitz, M. J., Gibbs, A. J., Watson, L. and Zurcher, E. J. (eds.) (1996 onwards). 'Plant Viruses Online: Descriptions and Lists from the VIDE Database. Version: 20th August 1996.'

Canto, T., MacFarlane, S. A., and Palukaitis, P. 2004. ORF6 of Tobacco mosaic virus is a determinant of viral pathogenicity in Nicotiana benthamiana. J. Gen. Virol. 85:3123-3133.

Drugeon, G., Urcuqui-Inchima, S., Milner, M., Kadare, G., Valle, R. P. C. Voyatzakis, A., Haenni, A., and Schirawski, J. 1999. The strategies of plant virus gene expression: Models of economy. Plant Sci. 148:77-88.

Fütterer, J., Potrykus, I., Bao, Y., Li, L., Burns, T. M., Hull, R., Hohn, T. 1996. Position-dependent ATT initiation during plant pararetrovirus rice tungro bacilliform virus translation. J. Virol. 70:2999-3010. 
Hacker, D. L., Petty, D. T., Wei, N., and Morris, T. J. 1992. Turnip crinkle virus genes required for RNA replication and virus movement. Virology 186:1-8.

Heaton, L. A., and Laakso, M. M. 1995. Several symptom-modulating mutations in the coat protein of turnip crinkle carmovirus result in particles with aberrant conformational properties. J. Gen. Virol. 76:225-230.

Heaton, L. A., Lee, T. C., and Morris, T. J. 1991. Point mutations in the turnip crinkle virus capsid protein affect the symptoms expressed by Nicotiana benthamiana. Virology 183:143-150.

Huang, M., Koh, D. C., Weng, L. J., Chang, M. L., Yap, Y. K., Zhang, L., and Wong, S. M. 2000. Complete nucleotide sequence and genome organization of hibiscus chlorotic ringspot virus, a new member of the genus Carmovirus: Evidence for the presence and expression of two novel open reading frames. J. Virol. 74:3149-3155.

Kozak, M. 2002. Pushing the limits of the scanning mechanism for initiation of translation. Gene 299:1-34.

Krishnamurthy, K., Heppler, M., Mitra, R., Blancaflor, E., Payton, M. Nelson, R. S., and Verchot-Lubicz, J. 2003. The Potato virus X TGBp3 protein associates with the ER network for virus cell-to-cell movement. Virology 309:135-151.

Krogh, A., Larsson, B., von Heijne, G., and Sonnhammer, E. L. 2001. Predicting transmembrane protein topology with a hidden Markov model: Application to complete genomes. J. Mol. Biol. 305:567-80.

Laemmli, U. K. 1970. Cleavage of structural proteins during the assembly of the head of bacteriophage T4. Nature 227:680-885.

Li, W. M., and Wong, S. M. 2006. Analyses of subgenomic promoters of Hibiscus chlorotic ringspot virus and demonstration of 5 ' untranslated region and 3'-terminal sequences functioning as subgenomic promoters. J. Virol. 80:3395-3405.

Li, W. Z., Qu, F., and Morris, T. J. 1998. Cell-to-cell movement of turnip crinkle virus is controlled by two small open reading frames that function in trans. Virology 244:405-416.

Liang, X. Z., Ding, S. W., and Wong, S. M. 2002a. Development of a kenaf (Hibiscus cannabinus L.) protoplasts system for replication study of hibiscus chlorotic ringspot virus. Plant Cell Rep. 20:982-986.

Liang, X. Z., Lucy, A. P., Ding, S. W., and Wong, S. M. 2002b. The p23 protein of Hibiscus chlorotic ringspot virus is indispensable for hostspecific replication. J. Virol. 76:12312-12319.

Lukhovitskaya, N. I., Yelina, N. E., Zamyatnin, A. A., Jr, Schepetilnikov, M. V., Solovyev, A. G., Sandgren, M., Morozov, S. Yu., Valkonen, J. P. T., and Savenkov, E. I. 2005. Expression, localization and effects on virulence of the cysteine-rich $8 \mathrm{kDa}$ protein of Potato mop-top virus. J. Gen. Virol. 86:2879-2889.

Meng, C.Y., Chen, J., Peng, J. R., and Wong, S. M. 2006. Host-induced avirulence of Hibiscus chlorotic ringspot virus mutants correlates with reduced gene silencing suppression activity. J. Gen. Virol. 87:451-459.

Neeleman, L., van der Kuyl, A. C., and Bol, J. F. 1991. Role of alfalfa mosaic virus coat protein gene in symptom formation. Virology 181:687-693.

Neeleman, L., Van Der Vossen, E. A. G., and Bol, J. F. 1993. Infection of tobacco with alfalfa mosaic virus cDNAs sheds light on function of the coat protein. Virology 196:883-887.

Omarov, R. T., Qi, D., and Scholthof, K.-B. G. 2005. The capsid protein of satellite Panicum Mosaic Virus contributes to systemic invasion and interacts with its helper virus. J. Virol. 79:9756-9764.

Petty, I. T., Hunter, B. G., Wei, N., and Jackson, A. O. 1989. Infectious barley stripe mosaic virus RNA transcribed in vitro from full-length genomic cDNA clones. Virology 171:342-349.

Rajendran, K. S., J. Pogany, and P. D. Nagy. 2002. Comparison of turnip crinkle virus RNA-dependent RNA polymerase preparations expressed in Escherichia coli or derived from infected plants. J. Virol. 76:17071717.

Rao, A. L. N., and Grantham, G. 1995. Biological significance of the seven amino-terminal basic residues of brome mosaic virus coat protein. Virology 211:42-52.

Schaad, M. C., Jensen, P. E., and Carrington, J. C. 1997. Formation of plant RNA virus replication complexes on membranes: Role of an endoplasmic reticulum-targeted viral protein. EMBO (Eur. Mol. Biol. Organ.) J. 16:4049-4059.

Schepetilnikov, M. V., Manske, U., Solovyev, A. G., Zamyatnin Jr, A. A., Schiemann, J., and Morozov, S. Yu. 2005. The hydrophobic segment of Potato virus $X$ TGBp3 is a major determinant of the protein intracellular trafficking. J. Gen. Virol. 86:2379-2391.

Shintaku, M. H., Zhang, L., and Palukaitis, P. 1992. A single amino acid substitution in the coat protein of cucumber mosaic virus induces chlorosis in tobacco. Plant Cell 4:751-757.

Shirako, Y. 1998. Non-AUG translation initiation in a plant RNA virus: A 40-amino-acid extension is added to the $\mathrm{N}$ terminus of the soil-borne wheat mosaic virus capsid protein. J. Virol. 72:1677-1682.

Suzuki, M., Kuwata, S., Masuta, C., and Takanami, Y. 1995. Point mutations in the coat protein of cucumber mosaic virus affect symptom expression and virion accumulation in tobacco. J. Gen. Virol. 76:17911799

Tamai, A., and Meshi, T. 2001a. Tobamoviral movement protein transiently expressed in a single epidermal cell functions beyond multiple plasmodesmata and spreads multicellularly in an infection-coupled manner. Mol. Plant-Microbe Interact. 14:126-134.

Tamai, A., and Meshi, T. 2001b. Cell-to-cell movement of Potato virus $X$ : The role of $\mathrm{p} 12$ and $\mathrm{p} 8$ encoded by the second and third open reading frames of the triple gene block. Mol. Plant-Microbe Interact. 14:11581167.

van der Kuyl, A. C., Neeleman, L., and Bol, J. F. 1991. Role of alfalfa mosaic virus coat protein in regulation of the balance between viral plus and minus strand synthesis. Virology 185:496-499.

White, K. A., Skuzeski, J. M., Li, W., Wei, N., and Morris, T. J. 1995. Immunodetection, expression strategy and complementation of turnip crinkle virus p28 and p88 replication components. Virology 211:525-534.

Yang, Y. N., Li, R. G., and Qi, M. 2000. In vivo analysis of plant promoters and transcription factors by agroinfiltration of tobacco leaves. Plant Sci. 122:101-108.

You, X. J., Kim, J. W., Sturat, G. W., and Bozarth, R. F. 1995. The nucleotide sequence of cowpea mottle virus and its assignment to the genus Carmovirus. J. Gen. Virol. 76:2841-2845.

\section{AUTHOR-RECOMMENDED INTERNET RESOURCES}

Weizmann Institute of Science Bioinformatics and Biological Computing website: bioinformatics.weizmann.ac.il,

Center for Biological Sequence Analysis TMHMM server version 2.0: www.cbs.dtu.dk/services/TMHMM

As originally published, the affiliations for the first two authors were incorrect. The correct affiliations for T. Zhou and Z. F. Fan are the Department of Plant Pathology and State Key Laboratory of Agrobiotechnology, China Agricultural University, Beijing 100094, China. This research was also partly supported by the National Basic Research Program of China (\#2006CB101900). This change was made October 5, 2006. 